\title{
Prognostic significance of tumor laterality in advanced ovarian cancer
}

\author{
Yuki Yamada, MD, PhD ${ }^{1}$, Seiji Mabuchi, MD, PhD ${ }^{1,2}$, Naoki Kawahara, MD, PhD ${ }^{1}$, Ryuji Kawaguchi, MD, PhD \\ ${ }^{1}$ Department of Obstetrics and Gynecology, Nara Medical University, Kashihara; ${ }^{2}$ Department of Gynecology, Osaka International Cancer Institute, \\ Osaka, Japan
}

\section{Objective}

This study aimed to investigate the effect of incorporating tumor laterality into the International Federation of Gynecology and Obstetrics (FIGO) staging system for advanced ovarian cancer.

\section{Methods}

The clinical data of 131 patients with advanced ovarian cancer treated between 2008 and 2018 were retrospectively reviewed. To investigate the prognostic significance of tumor laterality, we divided the patients into unilateral and bilateral groups. The prognostic significance of tumor laterality (bilateral vs. unilateral) was evaluated using univariate and multivariate analyses. The effect of incorporating tumor laterality into the FIGO staging system to predict survival outcomes was evaluated using the Kaplan-Meier method.

\section{Results}

Both overall survival (OS) and progression-free survival (PFS) were longer in the unilateral group than in the bilateral group. Multivariate analysis demonstrated that tumor laterality was an independent predictor of OS (hazard ratio, 1.75; confidence interval, 1.05-2.92; $P=0.032$ ). In patients with stage III disease, the bilateral group had a shorter OS than the unilateral group, but it was comparable to the OS in stage IV patients $(P=0.354)$. The incorporation of tumor laterality into the FIGO staging system improved the stratification of survival probabilities.

\section{Conclusion}

Tumor laterality can be an independent prognostic factor in patients with advanced ovarian cancer. The incorporation of tumor laterality may improve the predictive performance of the FIGO staging system in patients with advanced ovarian cancer.

Keywords: FIGO staging system; Ovarian cancer; Prognosis; Risk factors; Tumor laterality

\section{Introduction}

The most recent global statistics estimated that 295,414 women were newly diagnosed with epithelial ovarian cancer, and 184,799 would die. Such a high mortality rate is primarily attributed to the fact that early disease produces nonspecific symptoms, leading to delayed diagnoses until the late stages, and more than $75 \%$ of ovarian cancer cases have been diagnosed as advanced-stage diseases, which are difficult to treat effectively [1].

In malignant diseases, the staging system is important because it defines prognosis and is a guiding tool for treatment options. Moreover, by employing a universally accepted staging system, the efficacy of anticancer treatments can be evaluated or compared with that reported in different trials. The International Federation of Gynecology and Obstetrics (FIGO) staging system is a widely accepted staging method

Received: 2021.06.04. Revised: 2021.07.17. Accepted: 2021.08.10. Corresponding author: Seiji Mabuchi, MD, PhD

Department of Gynecology, Osaka International Cancer Institute, 3-1-69 Otemae, Chuo-ku, Osaka 541-8567, Japan

E-mail: seiji.mabuchi@oici.jp

https://orcid.org/0000-0003-1803-1166

Articles published in Obstet Gynecol Sci are open-access, distributed under the terms of the Creative Commons Attribution Non-Commercial License (http://creativecommons. org/licenses/by-nc/3.0/) which permits unrestricted non-commercial use, distribution, and reproduction in any medium, provided the original work is properly cited.

Copyright $\odot 2021$ Korean Society of Obstetrics and Gynecology 


\section{Obstetrics \& Gynecology Science}

Yuki Yamada, et al. Tumor laterality in ovarian cancer

for gynecological malignancies and estimates the survival of ovarian cancer patients as follows: $89 \%$ for stage I, $71 \%$ for stage II, $41 \%$ for stage III, and $20 \%$ for stage IV [2]. Although the FIGO staging system has been periodically revised to more accurately reflect patient prognosis based on the latest findings, its ability to estimate survival is far from optimal, especially in patients with advanced-stage ovarian cancer.

The prognostic factors for patients with advanced-stage ovarian cancer have been intensively investigated, and the histological subtype, presence and extent of lymph node metastasis, presence of ascites, completeness of surgical resection, platinum-sensitive disease, and BRCA status are well-known significant prognostic factors $[3,4]$. However, the ability of these conventional risk factors to predict recurrence and estimate survival is insufficient.

Previous studies have suggested that tumor laterality in paired organs is predictive of patient survival, and patients with left testicular cancer, right lung cancer, and right breast cancer showed significantly better survival than those with contralateral disease [5-11]. So far, three studies have investigated the prognostic significance of tumor laterality in patients with ovarian cancer [11-13] but have produced conflicting results. At this point, in ovarian cancer, tumor laterality has only been included in the FIGO staging system in stage I patients: stage IA, tumor limited to one ovary, and stage IB, tumor involving both ovaries. In stage II or greater disease, tumor laterality is not included in the staging system.

In this study, we investigated the prognostic significance of tumor laterality in patients with advanced-stage ovarian cancer. We also investigated the effect of incorporating tumor laterality into the FIGO staging system for prognostic prediction.

\section{Materials and methods}

\section{Patients}

This retrospective study was approved by the institutional review board of Nara Medical University (approval number: 2879). This study included patients who were clinically diagnosed with FIGO 2014 stage III or IV primary invasive ovarian cancer between January 2008 and December 2018. Among the 131 patients included in the current study, 52 underwent comprehensive surgical staging. The remaining 79 patients underwent comprehensive staging surgery or exploratory surgery (laparotomy or laparoscopy) and were clinically staged according to the findings of preoperative computed tomography. Patients with concurrent primary malignancies of other organs or who were not followed up were excluded.

A comprehensive review of the medical records was performed, and the following variables were obtained: age at diagnosis, body mass index (BMI), pretreatment plasma cancer antigen 125 (CA125) level, imaging results (computed tomography of the chest and abdomen or magnetic resonance imaging of the pelvis), surgical details, pathological findings, adjuvant treatment information, date of disease progression or death, and patient status at the recent follow-up. Ascites formation was defined as the presence of ascitic fluid beyond the small pelvic cavity, as assessed by computed tomography.

\section{Treatments}

At our institution, patients were assigned to a treatment regimen after a multidisciplinary discussion among gynecologists, radiologists, and pathologists. Patients were primarily treated with primary debulking surgery (PDS) followed by adjuvant chemotherapy, neoadjuvant chemotherapy (NACT) followed by interval debulking surgery (IDS), or chemotherapy alone. Prior to chemotherapy, the patients were histologically diagnosed through exploratory laparotomy. Recurrence was treated according to the Japan Society of Gynecologic Oncology guidelines [14]. Since January 2014, some patients have been treated with chemotherapy, including bevacizumab. None of the patients were treated with a polyadenosine diphosphate-ribose polymerase (PARP) inhibitor.

\section{Statistical analysis}

Statistical analyses were conducted using the SPSS software (version 22.0, IBM corp., Armonk, NY, USA). Clinicopathological characteristics were compared using the $\chi^{2}$-test, Fisher's exact test, and Mann-Whitney U-test, as appropriate. Progression-free survival (PFS) was defined as the duration from the first day of treatment to the detection of tumor progression or death from any cause. Overall survival (OS) was defined as the time from the first day of treatment to the date of death from any cause. Survival data were plotted as Kaplan-Meier curves, and significant differences were determined using the log-rank test. $P$-values were two-sided, and a $P$-value $<0.05$ indicated statistical significance. Univariate analyses were performed by comparing the Kaplan-Meier curves. Cox proportional hazard regression analysis was performed to identify the independent predictors of survival. 


\section{Obstetrics \& Gynecology Science}

Vol. 64, No. 6, 2021

\section{Results}

\section{Patients}

A total of 131 patients were included in the study. The median follow-up period was 34.6 months for all patients. The clinicopathological characteristics of the patients are summarized in Table 1. PDS was performed in 63 patients (48.1\%). NACT followed by IDS treatment was performed in 63 patients $(48.1 \%)$. Five patients were treated with chemotherapy alone. The primary chemotherapy regimen was a paclitaxel-carboplatin combination, and 19 patients received chemotherapy with bevacizumab. The most prevalent histological type was high-grade serous carcinoma (HGSC) (70/131, 42.7\%), followed by endometrioid carcinoma $(21 / 131,12.8 \%)$. Seventy-three patients had unilateral ovarian cancer (unilateral group), and 58 patients had bilateral ovarian cancer (bilateral group).

The relationships between tumor laterality and clinical characteristics are summarized in Table 1. There were no differences in age, BMI, FIGO stage, CA125 level, and ascites formation between the two groups. However, the number of patients with serous ovarian cancer and those with residual tumors after PDS was significantly higher in the bilateral group than in the unilateral group.

\section{Prognostic significance of tumor laterality}

\section{1) Right versus left}

In the unilateral group, 40 patients had right-sided ovarian cancer, and 33 patients had left-sided ovarian cancer. The clinicopathological characteristics of patients with right-sided versus left-sided ovarian cancer according to the site of origin are listed in Supplementary Table 1. There were no differences in patient characteristics, except for the tumor diameter. There was no significant difference in OS and PFS according to tumor laterality (OS, $P=0.218$; PFS, $P=0.604$ ) (Supplementary Fig. 1).

Table 1. Clinicopathological characteristics of the patients included in the current study

\begin{tabular}{|c|c|c|c|}
\hline & Unilateral group $(n=73)$ & Bilateral group $(n=58)$ & $P$-value \\
\hline Age (yr) & $61.0(38-90)$ & $60.0(16-77)$ & 0.323 \\
\hline $\operatorname{BMI}\left(\mathrm{kg} / \mathrm{m}^{2}\right)$ & $22.2(16.3-35.2)$ & $21.9(13.3-31.6)$ & 0.993 \\
\hline FIGO stage & & & 0.216 \\
\hline III & $50(68.5)$ & $35(60.3)$ & \\
\hline IV & $23(31.5)$ & $23(39.7)$ & \\
\hline Histologic subtype & & & $<0.001$ \\
\hline Non-serous & $44(60.3)$ & $17(29.3)$ & \\
\hline Serous & $29(39.7)$ & $41(70.7)$ & \\
\hline Primary treatment & & & $<0.001$ \\
\hline PDS+chemothrapy & $45(61.6)$ & $18(31.0)$ & \\
\hline NACT+IDS & $28(38.4)$ & $35(60.4)$ & \\
\hline Chemotherapy alone & 0 & $5(8.6)$ & \\
\hline CA125 & $437(22-18,338)$ & $582(11-17,925)$ & 0.270 \\
\hline Ascites formation & & & 0.268 \\
\hline No & $21(28.8)$ & $13(22.4)$ & \\
\hline Yes & $52(71.2)$ & $45(77.6)$ & \\
\hline Residual tumor & & & $<0.001$ \\
\hline No & $21(28.8)$ & $2(3.4)$ & \\
\hline Yes & $52(71.2)$ & $56(96.6)$ & \\
\hline Tumor diameter (mm) & $100(34-335)$ & $77(20-166)$ & 0.014 \\
\hline
\end{tabular}

Values are presented as median (range) or number (\%).

BMI, body mass index; FIGO, the international Federation of Gynecology and Obstetrics; PDS, primary debulking surgery; NACT, neoadjuvant chemotherapy; IDS, interval debulking surgery; CA125, cancer antigen 125. 


\section{Obstetrics \& Gynecology Science}

Yuki Yamada, et al. Tumor laterality in ovarian cancer

2) Unilateral versus bilateral

To investigate the prognostic significance of tumor laterality, we divided the patients into unilateral and bilateral groups.
The survival outcomes of the unilateral and bilateral groups are presented in Fig. 1. Both OS and PFS were longer in the unilateral group than in the bilateral group (OS, $P=0.004$;
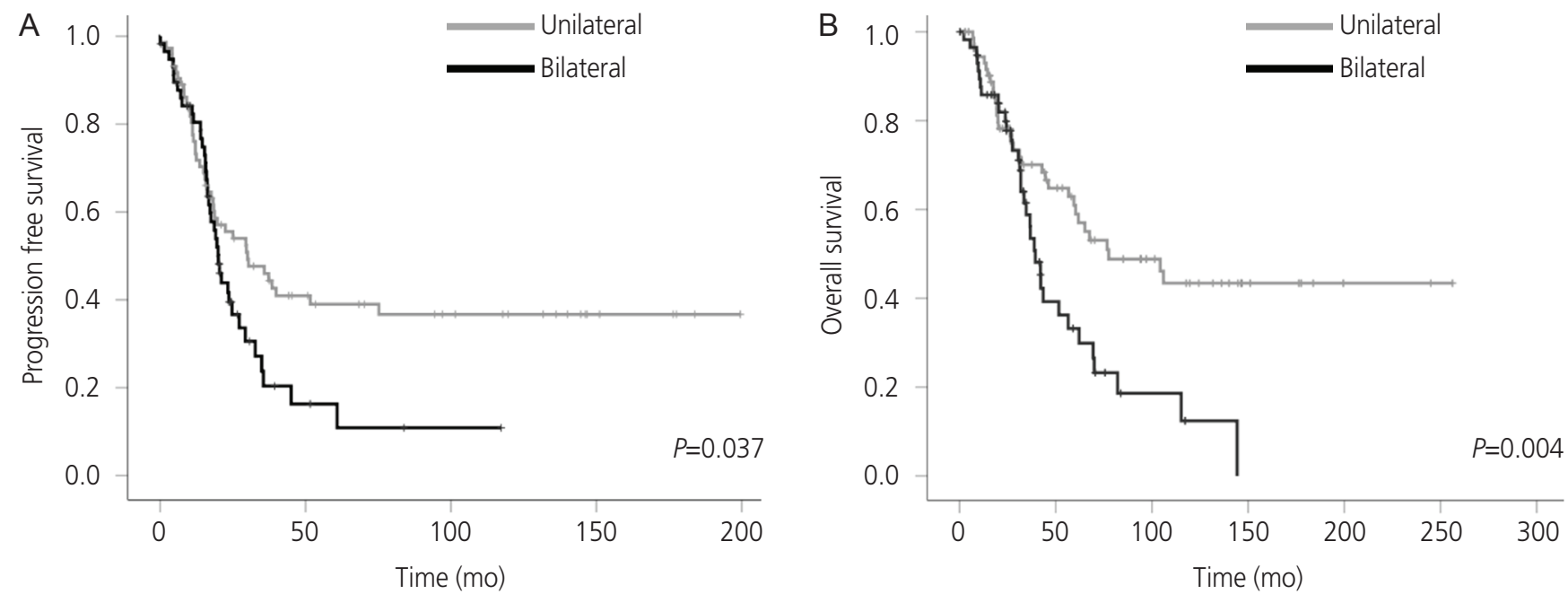

Fig. 1. Survival of patients with advanced ovarian cancer (stage III-IV) according to tumor laterality (unilateral vs. bilateral). Kaplan-Meier estimates of (A) progression-free survival (PFS) and (B) overall survival (OS). PFS and OS were significantly longer in the unilateral group than in the bilateral group.

Table 2. Univariate and multivariate analysis of prognostic factors in stage III-IV ovarian cancer

\begin{tabular}{|c|c|c|c|c|c|c|c|c|}
\hline & & \multirow{2}{*}{ Patients } & \multicolumn{3}{|c|}{ Univariate analysis } & \multicolumn{3}{|c|}{ Multivariate analysis } \\
\hline & & & Hazard ratio & $95 \% \mathrm{Cl}$ & $P$-value & Hazard ratio & $95 \% \mathrm{Cl}$ & $P$-value \\
\hline \multirow[t]{2}{*}{ Age (yr) } & $<55$ & 39 & Ref & & & & & \\
\hline & $\geq 55$ & 92 & 0.454 & $0.745-1.932$ & 0.355 & & & \\
\hline \multirow[t]{2}{*}{ BMI $\left(k g / m^{2}\right)$} & $<18.5$ & 25 & Ref & & & & & \\
\hline & $\geq 18.5$ & 106 & 1.648 & $0.910-2.985$ & 0.096 & & & \\
\hline \multirow[t]{2}{*}{ Laterality } & Unilateral & 73 & Ref & & & Ref & & \\
\hline & Bilateral & 58 & 1.601 & $1.026-2.498$ & 0.037 & 1.75 & $1.049-2.921$ & 0.032 \\
\hline \multirow[t]{2}{*}{ FIGO stage } & III & 85 & Ref & & & Ref & & \\
\hline & IV & 46 & 1.935 & $1.237-3.026$ & 0.003 & 1.834 & $1.112-30.24$ & 0.017 \\
\hline \multirow[t]{2}{*}{ Histologic subtype } & Non-serous & 61 & Ref & & & & & \\
\hline & Serous & 70 & 1.045 & $0.674-1.619$ & 0.845 & & & \\
\hline \multirow[t]{2}{*}{ CA125 } & $<274$ & 43 & Ref & & & & & \\
\hline & $\geq 274$ & 83 & 1.259 & $0.741-2.173$ & 0.367 & & & \\
\hline \multirow[t]{2}{*}{ Ascites formation } & No & 34 & Ref & & & & & \\
\hline & Yes & 97 & 1.49 & $0.799-2.782$ & 0.207 & & & \\
\hline \multirow[t]{2}{*}{ Residual tumor } & No & 23 & Ref & & & Ref & & \\
\hline & Yes & 108 & 2.543 & $1.159-5.580$ & 0.016 & 1.82 & $0.798-4.152$ & 0.155 \\
\hline \multirow[t]{2}{*}{ Tumor diameter (mm) } & $>50.5$ & 22 & Ref & & & & & \\
\hline & $\geq 50.5$ & 109 & 1.845 & $0.797-4.271$ & 0.383 & & & \\
\hline
\end{tabular}

$\mathrm{Cl}$, confidence interval; BMl; body mass index, FIGO; the international Federation of Gynecology and Obstetrics; CA125, cancer antigen 125. 


\section{Obstetrics \& Gynecology Science}

Vol. 64, No. 6, 2021

PFS, $P=0.037$ ).

The results of the univariate and multivariate Cox regression analyses for OS are shown in Table 2. The appropriate cutoff values for age, BMI, serum CA125 level, and tumor diameter were calculated from the receiver operating characteristic curve analysis (Supplementary Fig. 2). Univariate analysis revealed that bilateral tumors, FIGO stage IV, and residual tumors were associated with a shorter OS $(P<0.05)$.

These results were further demonstrated by multivariate analysis showing tumor laterality as an independent predictor of OS (hazard ratio [HR], 1.75; 95\% confidence interval
$[C I], 1.05$ to $2.92 ; P=0.032)$.

To exclude the possibility that insufficient surgical debulking was the sole reason for poor prognosis in the bilateral group, we further evaluated the prognostic significance of tumor laterality in stage III patients with residual tumor after PDS. There were 66 patients with residual tumors after primary surgery, with 33 patients each in the unilateral and bilateral groups. The OS was longer in the unilateral group than in the bilateral group ( $P=0.014)$ (Supplementary Fig. 3).
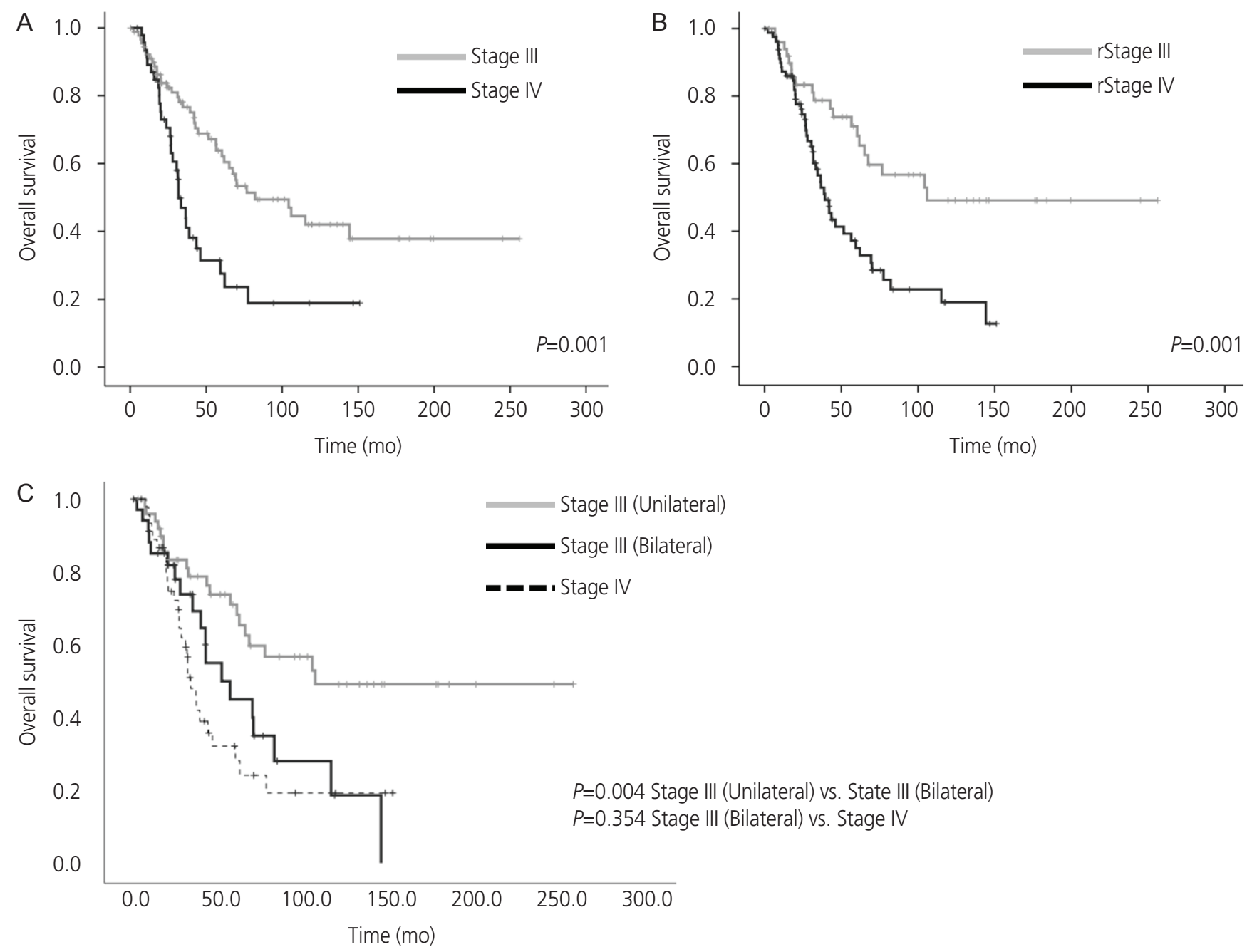

Fig. 2. Clinical implications of tumor laterality in patients with ovarian cancer according to the disease stage. (A) Incorporation of tumor laterality into the International Federation of Gynecology and Obstetrics (FIGO) staging system. Survival curves according to the stage grouping in the two systems. Figure (A, B) reflect the difference in distribution of stage III patients; the revised FIGO staging system widens the distance between the curves, thus better stratifying the survival probabilities. (C) Kaplan-Meier estimates of overall survival (OS) according to tumor laterality. The OS of the stage III bilateral group was significantly shorter than that of the stage III unilateral group $(P=0.004)$ and comparable to that of stage IV ovarian cancer patients $(P=0.354)$. 


\section{Obstetrics \& Gynecology Science}

Yuki Yamada, et al. Tumor laterality in ovarian cancer

\section{Incorporation of tumor laterality into the FIGO staging system}

According to the FIGO staging system, 85 (64.9\%) patients had stage III disease and 46 (35.1\%) had stage IV disease. As predicted, patients with stage IV disease exhibited a significantly shorter OS ( $P=0.001)$ (Fig. 2A).

We investigated whether the incorporation of tumor laterality into the FIGO staging system could improve prognostic predictions in patients with FIGO stage III and IV ovarian cancer. Among the stage III ovarian cancers, the OS of the unilateral group was longer than that of the bilateral group $(P=0.004)$. The OS of the bilateral group with stage III disease was comparable to that of stage IV disease $(P=0.354)$ (Fig. 2C). We then created a new FIGO staging system by incorporating tumor laterality. In the revised FIGO staging system, patients who had been diagnosed with stage III bilateral ovarian cancer in the original FIGO staging system were classified as stage IV. According to the revised FIGO staging system, 35 patients had bilateral ovarian cancer, 50 (38.2\%) had stage III disease and 81 (61.8\%) had stage IV disease. The OS of the patients is shown in (Fig. 2B). Survival curves according to the stage grouping in the two systems reflect the difference in distribution of stage III patients, and the revised FIGO staging system widens the distance between the curves, thus better stratifying the survival probabilities. The results of the univariate and multivariate Cox regression analyses for OS are shown in Supplementary Table 2. It was demonstrated that revised FIGO staging (stage III vs. stage IV) was an independent predictor of OS, and the HR was higher in the revised FIGO stage than in the original FIGO staging (HR: 2.119 vs. 1.834).

\section{Discussion}

In the current study, we showed that tumor laterality (bilateral vs. unilateral) was an independent predictor of recurrence and survival in patients with stage III-IV ovarian cancer. We also demonstrated that the incorporation of tumor laterality improved the predictive performance of the FIGO staging system in these patients.

The prognostic significance of tumor laterality has been investigated in various solid malignancies. In colorectal cancers, it has been reported that right-sided tumors have poorer survival than left-sided tumors [5-7]. Similarly, in breast cancer, several reports have suggested that left-sided breast cancers have poorer survival than right-sided tumors [8-10]. In ovarian cancer, two studies reported that patients with left-sided ovarian cancer had a more favorable prognosis than those with right-sided ovarian cancer, presumably because of flow pattern dynamics in the peritoneal cavity or differential flow patterns of the right and left ovarian veins $[11,12]$. In addition, one study reported that patients with bilateral ovarian cancer showed shorter survival than those with unilateral ovarian cancer [13]. However, these studies were reported more than a decade ago, and there have been no reports on the relationship between tumor laterality and prognosis under the current treatment. In the current study, as shown in Supplementary Fig. 1, patients with right-sided ovarian cancer showed slightly better survival than those with leftsided tumors. However, this difference was not statisti cally significant $(P=0.211)$. The prognostic significance of tumor laterality requires further investigation.

The results from our study are consistent with those of a previous study showing that patients with unilateral ovarian cancer showed better survival than those with bilateral ovarian cancer [13]. The precise reasons for this finding remain unknown; however, we hypothesized several. First, ovarian cancer involving bilateral ovaries may exhibit increased invasiveness to the surrounding gastrointestinal or genitourinary organs than that involving a unilateral ovary, leading to the formation of unresectable disseminations and a decreased complete surgery rate during PDS. Multiple randomized controlled clinical trials and observational studies have confirmed a survival benefit associated with complete cytoreduction at the time of PDS. Resection of gross disease improved both PFS and OS [15-18]. In our study, the number of patients with residual tumors was higher in the bilateral group. To exclude the possibility that insufficient surgical debulking was the sole reason for the poor prognosis in the bilateral group, we further evaluated the prognostic significance of tumor laterality in patients with residual tumors only (Supplementary Fig. 3). Even in cases of residual tumors, patients with bilateral ovarian cancer had a poorer prognosis than those with unilateral ovarian cancer. This result suggests that there should be reasons other than insufficient surgical debulking for the poor prognosis in the bilateral group. Second, considering the fact that bilateral ovarian cancer develops through synchronous tumorigenesis or metastasis from one side to the other, ovarian cancer involving bilateral ovaries may have more subclini- 


\section{Obstetrics \& Gynecology Science}

Vol. 64, No. 6, 2021

cal metastases to the lymph nodes or visceral organs than those involving a unilateral ovary. In colorectal or breast cancer, several investigations have suggested that the survival differences can be attributed to the differences in the molecular and clinical characteristics of right-sided and left-sided tumors [5-10]. In the current study, we did not perform such mechanistic investigations. Thus, the differences in characteristics of bilateral or unilateral ovarian cancer need to be further investigated clinically and preclinically in future studies.

The results of this study have important clinical implications. Although tumor laterality cannot be precisely determined with imaging studies alone, by evaluating preoperative imaging studies, it may be possible to identify advancedstage ovarian cancer patients who are at high risk of developing recurrence or exhibiting shorter survival.

Moreover, the incorporation of tumor laterality into the FIGO staging system of advanced ovarian cancer would allow additional risk stratification, which may enable physicians to provide more aggressive cytoreductive surgery, more aggressive adjuvant treatment, or an intensive post-treatment follow-up program for advanced ovarian cancer patients with bilateral ovarian cancer. In our study, two patients in whom complete resection could be achieved showed a favorable prognosis compared to patients in whom complete resection could not be achieved (5-year OS: $100 \%$ vs. $42.0 \%$ ) (data not shown). Thus, at this point, maximum effort to achieve complete resection should be made for patients with bilateral ovarian cancer.

The limitations of our study need to be addressed. First, our study was conducted at a single institution, and the number of study cases was small. In a previous study investigating the significance of tumor laterality in stage III-IV ovarian cancer patients, the number of patients in the bilateral group was greater than that observed in the unilateral group (22 vs. 68), which is different from the current study (Table 1). Thus, the significance of tumor laterality in patients with advanced ovarian cancer must be further evaluated in a larger study. Second, the number of patients who underwent complete surgery was larger in the unilateral group than in the bilateral group in our study, but the number of complete surgeries for bilateral ovarian cancer patients may increase in another institution in which PDS is performed more actively. Third, due to its retrospective nature, potential confounding biases, such as selection bias introduced during the surgical staging or the selection of adjuvant treatments, may have been missed during the analysis. Fourth, as this study was conducted over a long period, changes in the choice of adjuvant treatments, the mode of pretreatment workup, and/or diagnostic procedures, or improvements in surgical procedures may have affected the survival of patients. To eliminate these potential biases, prospective multi-institutional investigations are required.

In conclusion, we found that tumor laterality was an independent predictor of recurrence and survival in patients with advanced ovarian cancer. The incorporation of tumor laterality may improve the predictive performance of the FIGO staging system in patients with advanced ovarian cancer.

\section{Conflict of interest}

No potential conflict of interest relevant to this article was reported.

\section{Ethical approval}

The study protocol was approved by the Institutional Review Board of Nara Medical University (approval no. 2879). The study was performed in accordance with the principles of the Declaration of Helsinki.

\section{Patient consent}

Informed consent from each patient was obtained in the form of an opt-out system on the website.

\section{Funding information}

This research did not receive any specific grant from funding agencies in the public, commercial, or not-for-profit sectors.

\section{Supplementary material}

Supplementary Table 1, 2, Supplementary Fig. 1-3 associated with this article can be found online at https://doi. org/10.5468/ogs.21176. 


\section{Obstetrics \& Gynecology Science}

Yuki Yamada, et al. Tumor laterality in ovarian cancer

\section{References}

1. Bray F, Ferlay J, Soerjomataram I, Siegel RL, Torre LA, Jemal A. Global cancer statistics 2018: GLOBOCAN estimates of incidence and mortality worldwide for 36 cancers in 185 countries. CA Cancer J Clin 2018;68:394424.

2. Torre LA, Trabert $B$, DeSantis $C E$, Miller KD, Samimi $G$, Runowicz CD, et al. Ovarian cancer statistics, 2018. CA Cancer J Clin 2018;68:284-96.

3. Tan DS, Rothermundt C, Thomas K, Bancroft E, Eeles R, Shanley $S$, et al. "BRCAness" syndrome in ovarian cancer: a case-control study describing the clinical features and outcome of patients with epithelial ovarian cancer associated with BRCA1 and BRCA2 mutations. J Clin Oncol 2008;26:5530-6.

4. Gallagher DJ, Konner JA, Bell-McGuinn KM, Bhatia J, Sabbatini P, Aghajanian CA, et al. Survival in epithelial ovarian cancer: a multivariate analysis incorporating BRCA mutation status and platinum sensitivity. Ann Oncol 2011;22:1127-32.

5. Negri F, De Giorgi A, Gilli A, Azzoni C, Bottarelli L, Gnetti $L$, et al. Impact of laterality and mucinous histology on relapse-free and overall survival in a registry-based colon cancer series. Sci Rep 2019;9:3668.

6. Yahagi M, Okabayashi K, Hasegawa H, Tsuruta M, Kitagawa $Y$. The worse prognosis of right-sided compared with left-sided colon cancers: a systematic review and meta-analysis. J Gastrointest Surg 2016;20:648-55.

7. Brulé SY, Jonker DJ, Karapetis CS, O'Callaghan CJ, Moore MJ, Wong R, et al. Location of colon cancer (right-sided versus left-sided) as a prognostic factor and a predictor of benefit from cetuximab in NCIC CO.17. Eur J Cancer 2015;51:1405-14.

8. Barbara RC, Piotr R, Kornel B, Elżbieta Z, Danuta R, Eduardo N. Divergent impact of breast cancer laterality on clinicopathological, angiogenic, and hemostatic profiles: a potential role of tumor localization in future outcomes. J Clin Med 2020;9:1708.
9. Bao J, Yu KD, Jiang YZ, Shao ZM, Di GH. The effect of laterality and primary tumor site on cancer-specific mortality in breast cancer: a SEER population-based study. PLoS One 2014;9:e94815.

10. Weiss HA, Devesa SS, Brinton LA. Laterality of breast cancer in the United States. Cancer Causes Control 1996;7:539-43.

11. Roychoudhuri R, Putcha V, Møller H. Cancer and laterality: a study of the five major paired organs (UK). Cancer Causes Control 2006;17:655-62.

12. Meleka F, Rarla S. Variation of spread of ovarian malignancy according to site of origin. Gynecol Oncol 1975;3:108-13.

13. Weiss NS, Silverman DT. Laterality and prognosis in ovarian cancer. Obstet Gynecol 1977;49:421-3.

14. Komiyama S, Katabuchi H, Mikami M, Nagase S, Okamoto A, Ito K, et al. Japan Society of Gynecologic Oncology guidelines 2015 for the treatment of ovarian cancer including primary peritoneal cancer and fallopian tube cancer. Int J Clin Oncol 2016;21:435-46.

15. Chi DS, Eisenhauer EL, Zivanovic O, Sonoda Y, AbuRustum NR, Levine DA, et al. Improved progressionfree and overall survival in advanced ovarian cancer as a result of a change in surgical paradigm. Gynecol Oncol 2009;114:26-31.

16. Chang SJ, Hodeib M, Chang J, Bristow RE. Survival impact of complete cytoreduction to no gross residual disease for advanced-stage ovarian cancer: a meta-analysis. Gynecol Oncol 2013;130:493-8.

17. Bristow RE, Tomacruz RS, Armstrong DK, Trimble EL, Montz FJ. Survival effect of maximal cytoreductive surgery for advanced ovarian carcinoma during the platinum era: a meta-analysis. J Clin Oncol 2002;20:1248-59.

18. Eisenkop SM, Spirtos NM, Friedman RL, Lin WC, Pisani AL, Perticucci S. Relative influences of tumor volume before surgery and the cytoreductive outcome on survival for patients with advanced ovarian cancer: a prospective study. Gynecol Oncol 2003;90:390-6. 